\title{
INFERENCIA DE CONTEXTO EN APLICACIONES MÓVILES INTELIGENTES
}

\author{
Reiner Solís Villanueva, Marcelo Arakaki, Carlos Valverde
}

\begin{abstract}
Resumen
El presente artículo propone un modelo de un sistema basado en técnicas de inteligencia artificial para inferir el contexto del usuario y optimizar el desarrollo de aplicaciones dependientes del contexto en los dispositivos móviles. En el documento se analiza la viabilidad de la aplicación de las redes neuronales artificiales en la optimización de este tipo de aplicaciones; el objetivo es construir un marco general de inferencia alternativo a los ya existentes, con el fin de proporcionar una infraestructura básica para facilitar el desarrollo de aplicaciones dependientes del contexto (contextaware), mediante la integración de la información procedente del entorno.
\end{abstract}

Palabras clave: Aplicaciones informáticas dependientes del contexto / dispositivos móviles / redes neuronales artificiales.

IחדーRーA 


\section{Introducción}

Tanto en el Perú como en el mundo, en los próximos años, los dispositivos móviles se convertirán en herramientas de autoadaptación dinámica al contexto del usuario, por lo que el diseño de un sistema basado en técnicas de inteligencia artificial, para aplicaciones conscientes del entorno, no solo será un lujo sino una necesidad que permitirá facilitar y optimizar el desarrollo de aplicaciones en los dispositivos móviles.

El desarrollo de los actuales dispositivos móviles está cambiando el paradigma de la computación móvil y obligando a rediseñar las aplicaciones empresariales. Eric Schmidt, CEO de Google (2013), considera que el futuro de la informática se encuentra en los dispositivos móviles inteligentes y los centros de datos (data center), y que en los próximos años las tecnologías móviles continuará avanzando y los consumidores estarán expuestos a aplicaciones inimaginables hasta ahora.

El entorno adquiere vital importancia en el desarrollo de las actuales aplicaciones en tecnología de información. Según la empresa de investigación en tecnologías de la información Gartner Inc., una aplicación context-aware es un sistema capaz de extraer, interpretar y usar información contextual y adaptar su comportamiento de acuerdo al entorno. Es necesario involucrar estos aspectos y aplicar la analítica operacional predictiva para inferir el contexto mediante la aplicación de técnicas de inteligencia artificial de modelos comprobados, usando el contexto impuesto por el uso de dispositivos móviles.

La investigación propuesta se realizó teniendo como base el marco de inferencia de contexto básico desarrollado en los trabajos publicados por Jesús Díaz et al., Inteligencia ambiental en dispositivos móviles (2009); y Paolo Coppola et al., The context-aware browser (2010). Estos enfoques son el punto de partida para el desarrollo de un marco de inferencia alternativo de aplicaciones context-aware.

\section{Marco teórico}

\subsection{Dispositivos móviles inteligentes}

El desarrollo tecnológico y las innovaciones en los dispositivos móviles crecen rápidamente. La evolución del teléfono móvil del siglo XX al presente siglo ha demostrado que este dispositivo es capaz de convertirse en una herramienta autoadaptativa dinámica de contexto de acuerdo a las necesidades del usuario. Los avances respecto al hardware que componen estos dispositivos, tanto en la capacidad de procesamiento y en almacenamiento de datos, brinda un acercamiento a la capacidad de procesamiento de las computadoras personales, generando el nacimiento de un nuevo concepto: smartphone, un dispositivo móvil 
que sobrepasa la capacidad de realizar simplemente una llamada (Cyberstore 2009). Es posible que en un futuro este dispositivo pueda migrar a convertirse en el reemplazo de los ordenadores móviles y los ordenadores de mesa.

Últimamente, las características más resaltantes en la gama alta de los teléfonos móviles son las pantallas táctiles, GPS y el sensor de movimiento (acelerómetro). La pantalla táctil se ha vuelto indispensable en reemplazo de los teclados tradicionales (teclados qwerty) para una interacción más amigable entre el usuario y el dispositivo (Tsai et al., 2009). El GPS, sistema de posicionamiento global, informa sobre la ubicación del usuario a través de satélites, que proporcionan la latitud, longitud y altitud de un objeto (Oficina de Coordinación Nacional de Posicionamiento, Navegación y Cronometría por Satélite 2011). La presencia del sensor de movimiento o acelerómetro es cada vez más importante, especialmente en muchos de los modelos con pantalla táctil, como por ejemplo el iPhone 5; por ahora este sensor es usado con mayor incidencia por el mercado de videojuegos.

Todo hardware necesita de un software que lo gestione. Sin un sistema operativo adecuado se desperdicia la gran capacidad potencial que ofrecen los teléfonos móviles. Por ello, es importante reconocer el mercado actual de las plataformas disponibles en el mercado (Albeson, Collins y Robie 2010). Según la cuota de mercado del primer trimestre del año 2013, el sistema operativo Android tiene el $75 \%$ de la cuota de mercado mundial (figura 1).

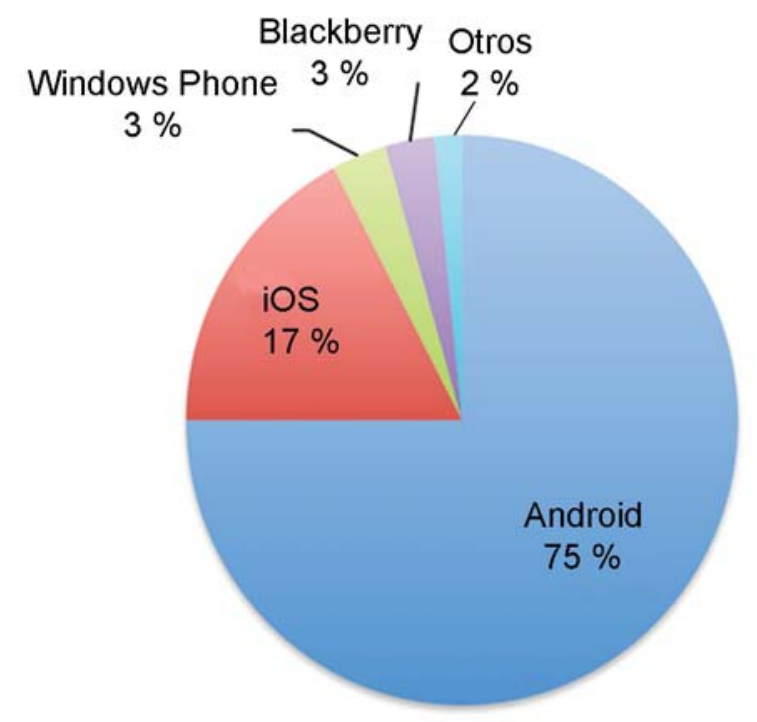

Figura 1. Cuota de mercado por sistema operativo

Fuente: IDC Worldwide Quarterly Mobile Phone Tracker, mayo del 2013. 
Al tener una mayor cuota de mercado hay un mayor número de desarrolladores para los sistemas operativos específicos, y como resultado una mayor disponibilidad de información acerca de este sistema y de soporte a la plataforma (Meier 2010).

\subsection{Inferencia de contextos}

El desarrollo de aplicaciones de entornos inteligentes o inteligencia ambiental, donde la tecnología se adapte al individuo y a su contexto de modo transparente y no intrusivo, genera una inteligencia ambiental (Carretero y Bermejo, 2005) en el que la interacción usuario-sistema se realiza de acuerdo a las necesidades del usuario, formando entornos invisibles pero integrados a los objetos cotidianos, desarrollando comportamientos predictivos a partir del conocimiento del contexto (contex aware) y de sus hábitos, y cuya importancia radica en los significativos cambios que implicará en la vida de las personas, facilitará sus labores diarias y se convertirá en una herramienta de apoyo a la gestión de la empresa, teniendo como centro de referencia al usuario.

Diferentes fuentes confirman la importancia de las investigaciones en contextaware y aplicaciones móviles, así como su relevancia futura. Recientemente, Gartner Inc. destacó a las aplicaciones centradas en dispositivos móviles e interfaces del Internet en las cosas dentro de las diez tecnologías estratégicas que tendrán un impacto significativo en los negocios el año 2013. El CEO de Google Eric Schmidt considera que el futuro de la informática se encuentra en las aplicaciones móviles inteligentes, y en los próximos años las tecnologías inteligentes continuarán avanzando y los consumidores serán expuestos al desarrollo de aplicaciones inimaginables hasta ahora.

En la actualidad, la visión de los modelos de computación está cambiando. Se abre la puerta al desarrollo de aplicaciones dinámicas más conscientes del entorno, a fin de integrarse en la vida del usuario. A raíz de esta visión, las plataformas de servicio de información se convierten en herramientas de autoadaptación dinámica, no solo busca la comodidad del usuario, sino que debe lograr que el entorno artificial sea lo menos intrusivo posible, de forma que el usuario solo sea consciente de sus ventajas pero no se considere invadido por la tecnología, que deben adaptarse a las necesidades cambiantes a partir de la información obtenida del entorno (Carretero y Bermejo, 2005), creando lo que se llama "Inteligencia ambiental".

En este contexto, el uso de diversas técnicas de inteligencia artificial, como redes neuronales artificiales, principios de sistemas basados en reglas, redes bayesianas y ontologías, pueden servir para construir un sistema que tenga la capacidad de inferir el contexto. Estos modelos han emergido como una potente herramienta 
para el modelado estadístico, orientada principalmente a la inferencia, predicción y reconocimiento de patrones, tanto en la vertiente de clasificación como de predicción.

En el caso de las redes neuronales estas poseen una serie de características admirables, tales como la habilidad para procesar datos con ruido o incompletos, la alta tolerancia a fallos que permite a la red operar satisfactoriamente con neuronas o conexiones dañadas y la capacidad de responder en tiempo real debido a su paralelismo inherente. Lo que se pretende con estos métodos es resolver el problema no solo de manera novedosa sino sobre todo tener soluciones efectivas.

\subsection{Redes neuronales artificiales}

Las redes neuronales artificiales forman parte de un conjunto de metodologías emergentes que se usan como soporte de trabajo de las organizaciones inteligentes y que actualmente tienen la denominación de análisis avanzado. Las redes neuronales artificiales no son programadas, aprenden a partir de ejemplos. Normalmente, a una red neuronal artificial se le presenta una serie de patrones de ejemplo a través de los cuales ellas deben aprender. Debido a que el aprendizaje es mediante ejemplos, las redes neuronales artificiales tienen un gran potencial para crear sistemas de computación que no necesitan ser programados. Esto supone un enfoque radicalmente distinto a los clásicos sistemas de software de desarrollo. En los programas de cómputo cada paso que el computador ejecuta debe ser previamente especificado por el programador, un solo proceso absorbe tiempo y recursos. Las redes neuronales comienzan con ejemplos de entradas y salidas y aprenden a producir la salida correcta para cada entrada. El enfoque de las redes neuronales consiste en que no requieren identificación de características, ni el desarrollo de algoritmos y programas para la resolución de problemas particulares. Sin embargo, presentan dos desventajas: el tiempo de aprendizaje de la red no puede ser conocido a priori, y el diseño de una red por prueba y error puede ser muy complejo. Esto quiere decir que mientras la red no haya concluido su proceso de aprendizaje no puede ser descartada (Isasi y Galván 2004).

Las redes neuronales artificiales codifican la información de manera distribuida. La información es almacenada en la red y es compartida por todas la unidades del proceso. Este esquema está en evidente contradicción con los esquemas tradicionales, donde los datos están almacenados en posiciones fijas de memoria. La información es almacenada en forma redundante y el resultado es un sistema tolerante a los fallos. Esto está en concordancia con la tolerancia a fallos de nuestros propios sistemas neuronales.

Actualmente existen variados modelos de redes neuronales artificiales que son usados en diversos campos de aplicación, destacando los modelos de Michie, 
Spiegelhalter y Taylor (1994), que es considerado como el estudio comparativo más completo entre redes neuronales artificiales y modelos estadísticos orientados a la clasificación (Sarle 1994), el modelo de la red de propagación hacia atrás (Rumelhart et al., 1986) y los mapas autoorganizados de Kohonen (1982). Estas estructuras pueden ser utilizadas en la clasificación y predicción del comportamiento de sistemas no lineales con información borrosa o incompleta, como es el caso de la gestión del riesgo. Un esquema de la red de propagación hacia atrás se muestra en la figura 2.

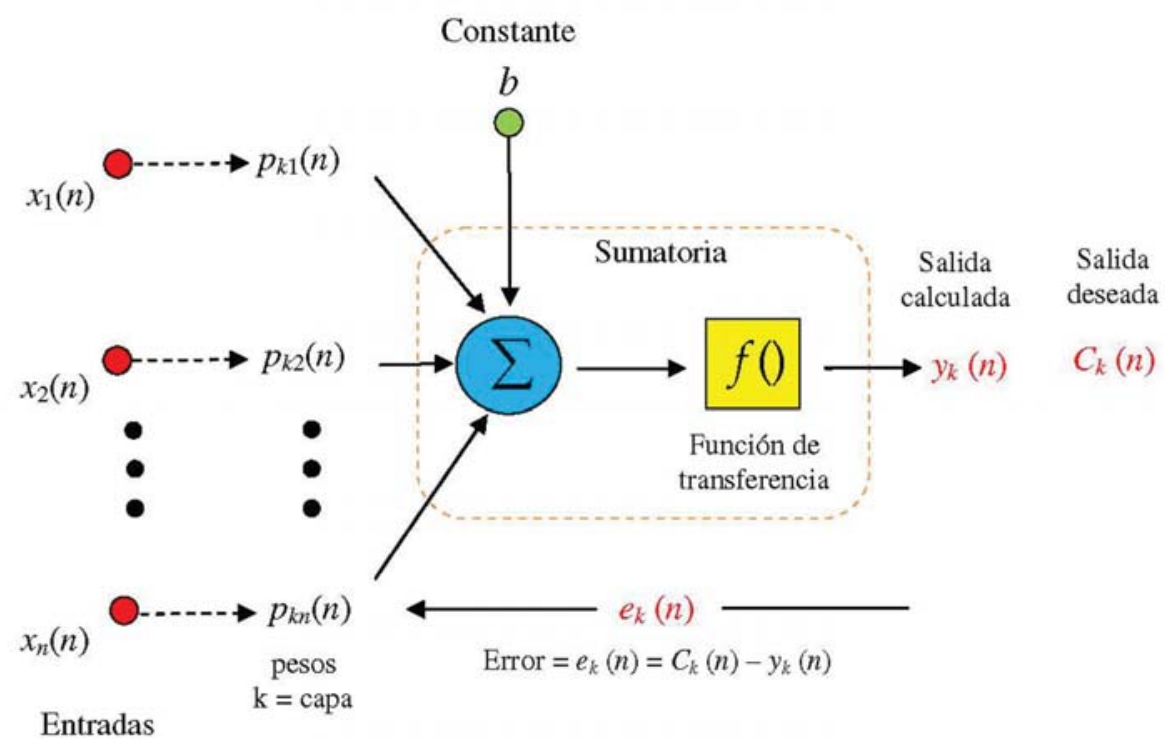

Figura 2. Estructura de la red neuronal en retropropagación

Fuente: Rumelhart, Hinton y Williams (1986).

\section{Descripción del problema}

A continuación se plantean las siguientes interrogantes asociadas al presente tema de investigación: ¿Es posible construir un modelo basado en técnicas de inteligencia artificial que permita inferir el contexto de un usuario de un dispositivo móvil?, ¿en qué medida el diseño e implementación de un sistema, basado en técnicas de inteligencia artificial, como herramienta estratégica para inferir el contexto del usuario, puede influir positivamente en la optimización del desarrollo de aplicaciones de entorno en los dispositivos móviles? 


\section{Metodología de solución}

\subsection{Contextos de análisis}

Considere un contexto general de un entorno cuyos sujetos tengan algunas características comunes y repetitivas. En este caso se define al usuario como un estudiante universitario propietario de un dispositivo móvil, y cuyo contexto de ubicación y actividad puede describirse dentro de determinados rangos y así lograr la definición de un contexto común de actuación, donde sea posible aprovechar el uso de los sensores de los dispositivos móviles inteligentes. Sin embargo, un estudiante promedio maneja una diversidad de contextos, identificados principalmente por la ubicación geográfica en la que se encuentra. Los estímulos captados por los sensores del dispositivo móvil pueden ser diferentes cuando el estudiante se encuentra en su casa o la universidad. En la investigación, se propone modelar la relación de los diversos estados de los sensores del dispositivo móvil con las actividades que realizan los estudiantes de la universidad en el día a día. Se dividió el contexto de actuación del usuario en dos grandes grupos:

a) Contexto hogar, el cual se encarga de inferir las actividades que realiza el estudiante en su hogar.

b) Contexto universidad, que se encarga de las actividades que realiza el estudiante dentro de la institución. Este contexto se divide en dos subcontextos:

i. Subcontexto para ambientes de estudio: instalaciones de la biblioteca de la universidad, los salones de clase, los laboratorios, los cubículos de estudio y los pasadizos de estos ambientes.

ii. Subcontexto para ambientes de descanso: patios de la universidad, jardines, cafetería y quioscos.

\subsection{Variables descriptoras del contexto}

Luego de analizar los diferentes sensores con los que vienen los dispositivos móviles, se ha optado por agrupar la funcionalidad de cada sensor en cuatro grupos: ubicación, intensidad lumínica, movimiento y tiempo (dimensión temporal). Considerando estos cuatro grupos, se propone un modelo que identifique la relación entre el estado de cada uno de estos grupos, entendidos como variables, y la probabilidad de que el usuario esté realizando una actividad genérica (comer, dormir, entre otras). 


\subsubsection{Ubicación}

Esta variable es un indicador de la ubicación específica donde se encuentra el individuo en uno de los contextos definidos. Por ejemplo, si el estudiante se encuentra en su casa, y específicamente en su dormitorio, esta variable puede tomar valor 1; o si está en su cocina puede tomar valor 2. En la concepción del modelo, esta variable requiere un filtro de los datos obtenidos por los sensores de ubicación, llámense GPS o WiFi, para conocer en qué ubicación se encuentra, además de una precisión inicial definida por el usuario.

\subsubsection{Intensidad Iumínica}

Esta variable evalúa la relación que puede tener la exposición del dispositivo móvil a la cantidad de luz que hay en el entorno del usuario y la actividad que está realizando, valiéndose del sensor de luz delantero del dispositivo móvil, y contribuye a identificar la actividad que realiza el usuario.

\subsubsection{Movimiento}

Para captar la cantidad de movimiento que el usuario aplica al dispositivo móvil se consideró al acelerómetro como el sensor fuente. También se evaluó el uso del giroscopio como sensor fuente; sin embargo, se descartó debido a que no aportaba información relevante sobre la actividad general que realiza el usuario.

\subsubsection{Tiempo}

El tiempo es una de las principales variables del modelo y los valores que toma van de 0 a 24 horas, y se tiene en cuenta los minutos como la parte decimal de la variable ( 1 minuto $=0.016666$ horas). Para obtener el tiempo se usa el reloj propio del dispositivo móvil, ya sea configurado por el usuario o por la operadora mediante la red móvil. No se toman en cuenta los segundos, ya que la mayoría de las actividades se rigen por horas y fracciones de horas traducidas a minutos.

\subsection{Actividades}

Las actividades que realizan los estudiantes se encontrarán relacionadas con los contextos donde estas se realicen. 


\subsubsection{Actividades realizadas en el contexto "casa'}

Para el contexto 'casa', se identificaron cuatro actividades generales, que servirán para identificar la influencia que tienen las variables anteriormente explicadas sobre ellas. Estas actividades son:
a) estudiar,
b) comer,
c) dormir, $y$
d) ocio.

\subsubsection{Actividades realizadas en el contexto 'universidad'}

Dentro de este contexto, las actividades realizadas se encontrarán asociadas a los dos subcontextos existentes, según el tipo de ambientes:

A. Actividades realizadas en el subcontexto 'ambientes de estudio'

Para este subcontexto se identificaron cuatro actividades comunes entre los estudiantes:
a) atender clases,
b) estudiar,
c) descansar, y
d) conversar.

B: Actividades realizadas en el subcontexto 'ambientes de descanso' Las actividades genéricas identificadas en este subcontexto son:
a) estudiar,
b) comer,
c) descansar,
d) caminar, $y$
e) ocio.

\section{Herramienta empleada en el modelo}

En los últimos años, se viene prestando un especial énfasis a ciertas técnicas matemáticas de autoadaptación a las relaciones que se tratan de modelar en diferentes investigaciones; una de las más importantes y mencionadas en la literatura son las redes neuronales artificiales. Una red neuronal es, a grandes rasgos, una herramienta matemática que busca emular la estructura y el comportamiento del cerebro humano, incluyendo un mecanismo de aprendizaje inducido por prueba y 
error. De esta forma, una red neuronal busca acoplar en su estructura las relaciones que pueden surgir en los estímulos (o variables de entrada) a los que se la expone y brindar una respuesta. Rumelhart, Hinton y Williams (1986) señalaron que una red neuronal valida si la respuesta brindada es aceptable y si no lo es ejecuta un mecanismo de corrección y adaptación que, dependiendo de la arquitectura de la red, puede estar basada en el error.

En este caso se emplea una red neuronal artificial de propagación hacia adelante que soporte el modelo en cada contexto y que bajo un plan de recopilación inicial de datos, particular para cada usuario, pueda aprender la rutina y evaluar la probabilidad de que en ese momento se esté realizando alguna de las actividades generales que se plantean en cada contexto. Para su aplicación, se consideraron las variables descriptoras del modelo como la capa de entrada de la red y las actividades como la capa de salida; entre estas capas hay un conjunto de capas escondidas que representan las relaciones existentes entre las variables y las actividades, y las conexiones entre capas representan el peso de cada relación por variable. Para poder emplear una aproximación de probabilidad para la salida de la red, se utilizó una función logística para la capa de salida, de manera que normalice los estímulos de respuesta a un rango de [0;1].

\section{Construcción del modelo}

El modelo general de desarrollo de la presente propuesta se muestra en la figura 3 , donde el contexto actual es establecido por los indicadores, determinados por los sensores del dispositivo móvil, que registran las actividades y acciones del usuario; esta base de datos constituye los datos de entrada al modelo. Previo a ello, sin embargo, hay que definir, mediante una etapa controladora, si la inferencia de contexto se realizará por las reglas definidas por el usuario o por predicción. Si todos los sensores del contexto actual están funcionando correctamente y definidas las reglas del usuario, la inferencia de contexto se realiza mediante un sistema de principios basado en reglas; sin embargo, si no están definidas la reglas del usuario, o los sensores del contexto actual del dispositivo móvil no están hábiles, la inferencia de contexto se realiza mediante predicción, basado en el aprendizaje del comportamiento cotidiano del usuario, empleando técnicas de inteligencia artificial. 


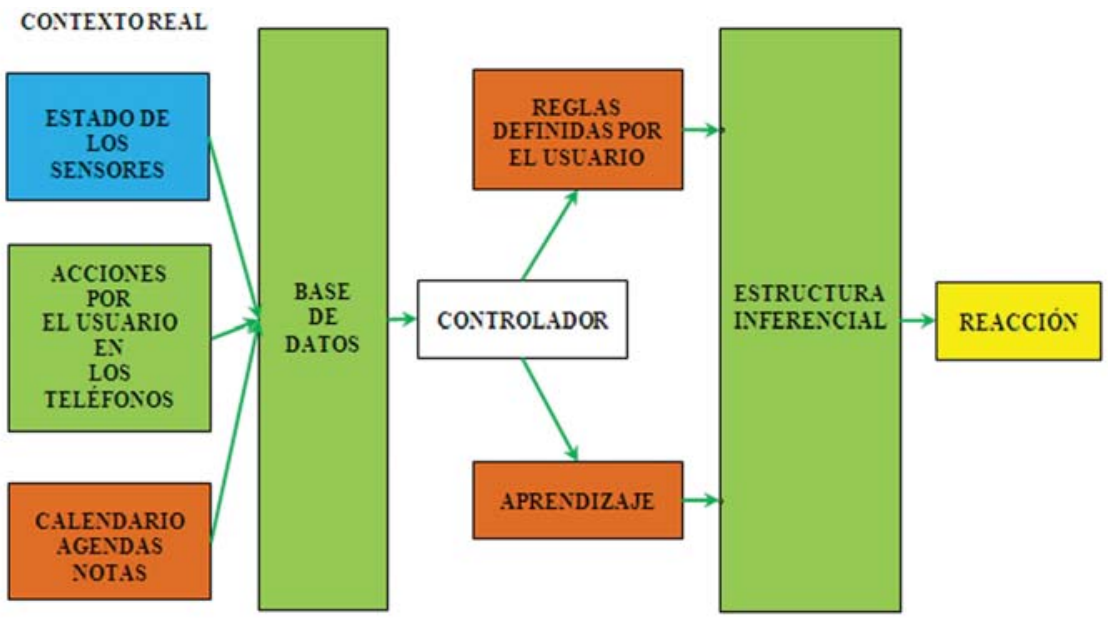

Figura 3. Modelo general propuesto para la inferencia de contexto

Elaboración propia.

La figura 4 presenta la propuesta de inferencia de contexto basado en una base de datos históricos del comportamiento cotidiano del usuario; sin embargo, este comportamiento cotidiano está limitado a un número de contextos predefinidos en el modelo a fin de que el sistema de inferencia de contexto pueda realizar la predicción.

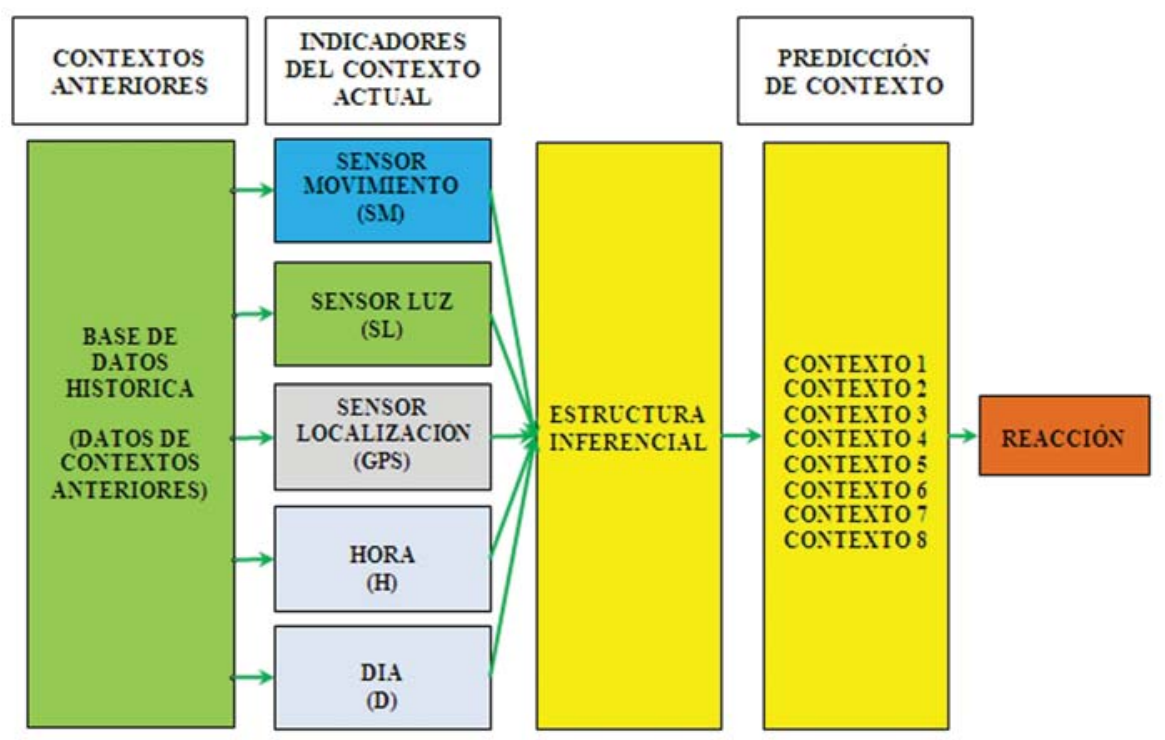

Figura 4. Modelo de inferencia de contexto

Elaboración propia. 
El modelo de inferencia de contexto por predicción se muestra en la figura 5, usando como herramienta de trabajo las redes neuronales artificiales, que es una técnica de inteligencia artificial de predicción incluso cuando los datos de entrada están incompletos. El modelo de red neuronal escogido es la red multicapa feed forward.

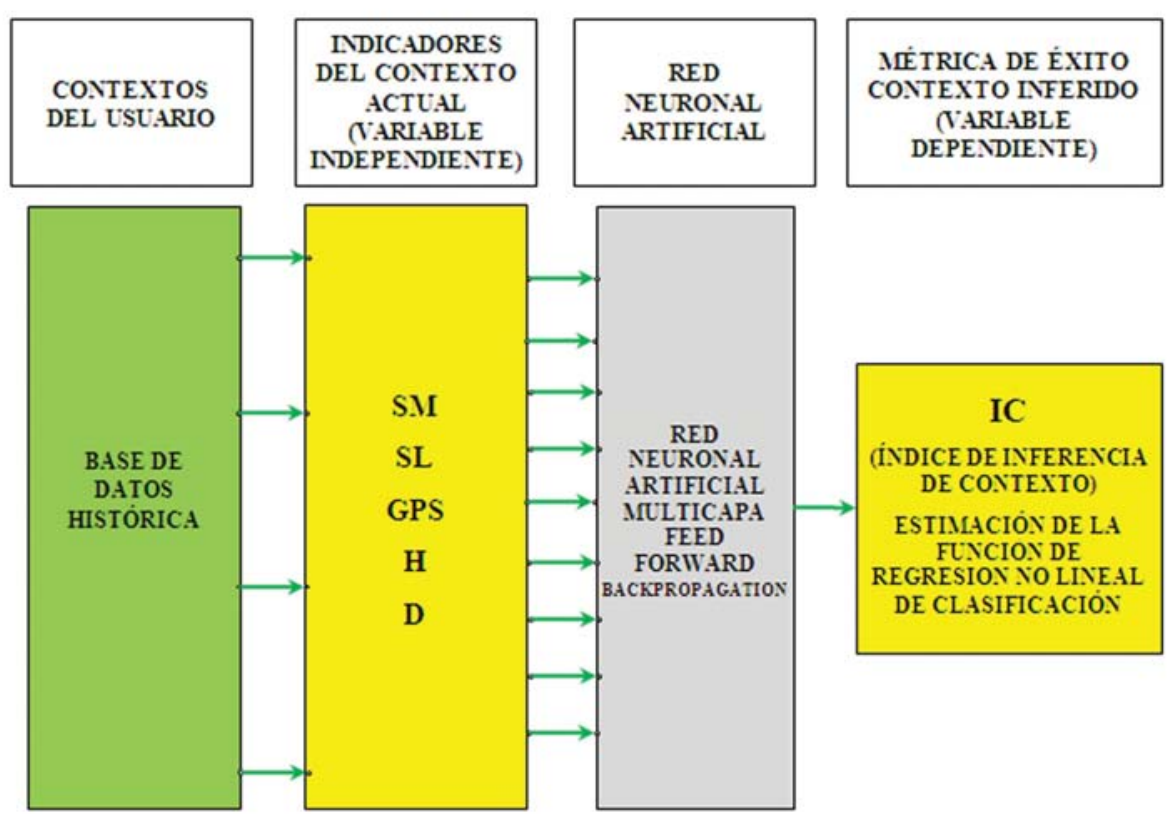

Figura 5. Modelo de inferencia de contexto por predicción

Elaboración propia.

El modelo de inferencia de contexto basado en el "principio de reglas" se aprecia en la figura 6, donde la inferencia de contexto se realiza por las reglas sugeridas al usuario. La regla es un conjunto de condiciones del entorno que se consideran importantes para el usuario y que establecen un número determinado de contextos relacionados a cada regla, que serán inferidos. 


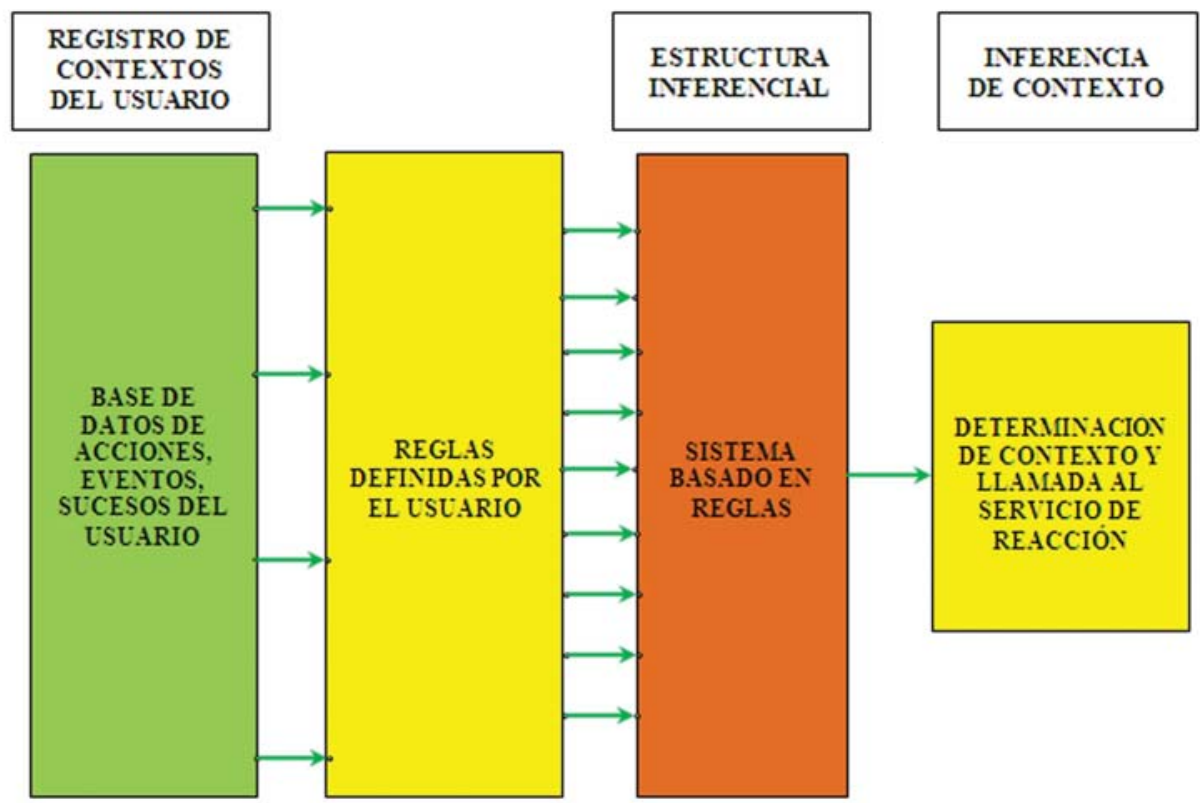

Figura 6. Inferencia de contexto. Sistema basado en reglas

Elaboración propia.

\section{Caso de estudio}

El caso realiza la inferencia de contexto para las actividades de un estudiante universitario que dispone de un smartphone (dispositivo móvil inteligente) y aprovecha la información de los sensores para crear una base de datos y determinar el comportamiento cotidiano del usuario.

\subsection{Variables de salida: contexto}

Las variables de salida dan como resultado la inferencia del contexto que realiza el modelo, a fin de inferir las actividades del estudiante, las cuales se agrupan de la siguiente forma:

a) Contexto casa.- En el presente caso de estudio la inferencia del contexto casa se limita a inferir la ubicación, no se evalúan las actividades dentro del contexto casa. 
b) Contexto universidad. - Se establecen las actividades que realiza dentro de la institución. Dentro de este contexto, las actividades realizadas se encontrarán asociadas a los dos subcontextos existentes: ambientes de estudio y ambientes de descanso.

Los valores que toma la variable de salida se presentan en la tabla 1.

\begin{tabular}{|l|l|l|}
\hline Rango & Contexto & Descripción \\
\hline 1 & Contexto 1 & Deshabilitado \\
\hline 2 & Contexto 2 & Estoy en casa \\
\hline 3 & Contexto 3 & Tránsito entre universidad y casa \\
\hline 4 & Contexto 4 & Universidad / Atiende clase \\
\hline 5 & Contexto 5 & Universidad / Estudia en la biblioteca \\
\hline 6 & Contexto 6 & Universidad / Descansa \\
\hline 7 & Contexto 7 & Universidad / Almuerza en cafetería \\
\hline 8 & Contexto 8 & Universidad / Camina \\
\hline
\end{tabular}

Tabla 1. Descripción de datos de las variables de salida

Elaboración propia.

\subsection{Variables de entrada proveniente de los sensores}

Se optó por agrupar la información de cada sensor en cuatro indicadores: ubicación, intensidad lumínica, movimiento y tiempo; los mismos que forman la base de datos para inferir el contexto.

a) Ubicación.- Especifica dónde se encuentra el individuo en uno de los contextos señalados por el usuario. El rango e identificación de cada lugar se muestra en la tabla 2.

b) Intensidad lumínica.- Evalúa la exposición del móvil a cantidad de luz que hay en el entorno del usuario y la actividad que está realizando. Los valores que toma esta variable son: Deshabilitado (1), Bajo (2), Medio (3) y Alto (4).

c) Movimiento.- Capta la cantidad de movimiento que el usuario aplica al dispositivo móvil, se considera el acelerómetro como el sensor fuente. Los valores que toma esta variable son: Deshabilitado (1), No movimiento (2) y En movimiento (3).

d) Tiempo.- Los valores que puede tomar van de 0 a 24 horas, y se tendrán en cuenta los minutos como la parte decimal de la variable (1 minuto $=0.016666$ horas). 
Los valores que toma la variable Día de la semana son: Domingo (1), Lunes (2), Martes (3), Miércoles (4), Jueves (5), Viernes (6), Sábado (7), conforme se describe en la tabla 2.

\begin{tabular}{|c|c|c|c|c|c|c|c|}
\hline \multicolumn{2}{|c|}{ Día de la semana } & \multicolumn{2}{|c|}{ Movimiento } & \multicolumn{2}{|c|}{ Intensidad lumínica } & \multicolumn{2}{|c|}{ Ubicación } \\
\hline Rango & Descripción & Rango & Descripción & Rango & Descripción & Rango & Descripción \\
\hline 1 & Domingo & 1 & Deshabilitado & 1 & Deshabilitado & 1 & Deshabilitado \\
\hline 2 & Lunes & 2 & No movimiento & 2 & Bajo & 2 & Casa \\
\hline 3 & Martes & 3 & En movimiento & 3 & Medio & 3 & $\begin{array}{l}\text { Exterior de universidad } \\
\text { y casa }\end{array}$ \\
\hline 4 & Miércoles & & & 4 & Alto & 4 & $\begin{array}{l}\text { Universidad / Puertas de } \\
\text { acceso, entrada y salida }\end{array}$ \\
\hline 5 & Jueves & & & & & 5 & Pabellón C \\
\hline 6 & Viernes & & & & & 6 & Pabellón CH / Cafetería \\
\hline \multirow[t]{14}{*}{7} & Sábado & & & & & 7 & Pabellón D \\
\hline & & & & & & 8 & Pabellón E \\
\hline & & & & & & 9 & Pabellón F \\
\hline & & & & & & 10 & Pabellón G \\
\hline & & & & & & 11 & Pabellón H \\
\hline & & & & & & 12 & Pabellón I \\
\hline & & & & & & 13 & Pabellón LL / Biblioteca \\
\hline & & & & & & 14 & Pabellón N \\
\hline & & & & & & 15 & Pabellón O \\
\hline & & & & & & 16 & Pabellón Q \\
\hline & & & & & & 17 & Pabellón R \\
\hline & & & & & & 18 & Pabellón S \\
\hline & & & & & & 19 & Pabellón W \\
\hline & & & & & & 20 & $\begin{array}{l}\text { Universidad / Patio, jar- } \\
\text { dines y quioscos }\end{array}$ \\
\hline
\end{tabular}

Elaboración propia.

Tabla 2. Descripción de datos. Variables de entrada 


\subsection{Base de datos}

Se considera un conjunto real de observaciones conformado por la información de los sensores del dispositivo móvil. Se logró tener la información de 13596 contextos, en cada contexto se identificaron cinco indicadores: Hora, Día de la Semana, Movimiento, Luz y ubicación, formando una base de 67980 datos, como se aprecia en la tabla 3.

\begin{tabular}{|c|c|c|c|}
\hline Actividades & $\begin{array}{c}\text { Número de } \\
\text { contextos }\end{array}$ & $\begin{array}{c}\text { Número de indicadores } \\
\text { por contexto }\end{array}$ & $\begin{array}{c}\text { Número de datos } \\
\text { por contexto }\end{array}$ \\
\hline 1 & 1942 & 5 & 9710 \\
\hline 2 & 1722 & 5 & 8610 \\
\hline 3 & 1505 & 5 & 7525 \\
\hline 4 & 900 & 5 & 4500 \\
\hline 5 & 1838 & 5 & 9190 \\
\hline 6 & 1828 & 5 & 9140 \\
\hline 7 & 1131 & 5 & 5655 \\
\hline 8 & 1319 & 5 & 6595 \\
\hline 9 & 1411 & 5 & 7055 \\
\hline Total datos de la red & $\mathbf{1 3 5 9 6}$ & $\mathbf{5}$ & $\mathbf{6 7 9 8 0}$ \\
\hline
\end{tabular}

Tabla 3. Base de datos

Elaboración propia

\subsection{Programación de la plataforma}

\subsubsection{Objetivos}

En esta investigación el principal objetivo fue el de desarrollar un prototipo de motor de captura de contextos haciendo uso del sistema operativo móvil Android.

\subsubsection{Herramientas y alcance}

Para su construcción, se implementó la captura de contextos definidos, de acuerdo a los valores de las variables en uso: ubicación, intensidad lumínica, movimiento y tiempo. Se utilizó el IDE Eclipse en la versión Indigo, con los plugin necesarios para programar en Android (ADT) y el SDK de Android Desarrollo. 
Para la programación del modelo se consideraron los dispositivos móviles trabajando con el sistema operativo Android 2.2. La programación para esta plataforma se realizó en código Java con Dalvik Virtual Machine. El desarrollo de la estructura de la aplicación siguió los parámetros bases de la investigación planteada por la Universidad Complutense de Madrid (Díaz et al., 2009). El esquema final desarrollado es el siguiente:

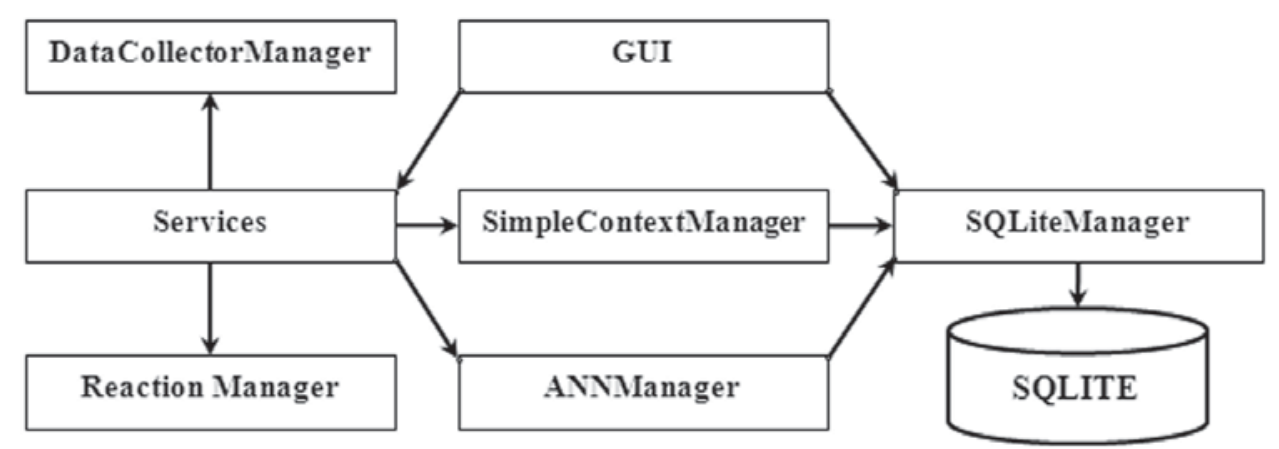

Figura 7. Esquema de desarrollo

Fuente: Díaz et al. (2009).

A. GUI.- Maneja todas las configuraciones que realiza el usuario para definir su regla/perfil. Una regla puede estar asociada a muchos contextos, pero cada contexto solo a una regla. Todo contexto debe estar asociado a un código de parámetros de contextos como el movimiento (clasificado con 1: Desactivado, 2: No movimiento y 3: En movimiento), Intensidad lumínica (1: Desactivado, 2: Bajo, 3: Medio, 4: Alto), ubicación (definido por el usuario) y el tiempo.

El usuario puede activar, desactivar, configurar y eliminar una regla que contiene la lista. Para crear una regla se selecciona el botón Nueva Regla; luego se define la regla que se va a ingresar. Posteriormente se configura el contexto, mediante la definición de un nuevo contexto y los parámetros del sensor. Se selecciona el lugar que se desea definir y se retorna la latitud y longitud automáticamente. Por último, se define el tiempo de inicio y el tiempo del fin y se seleccionan los días que estarán activos. 

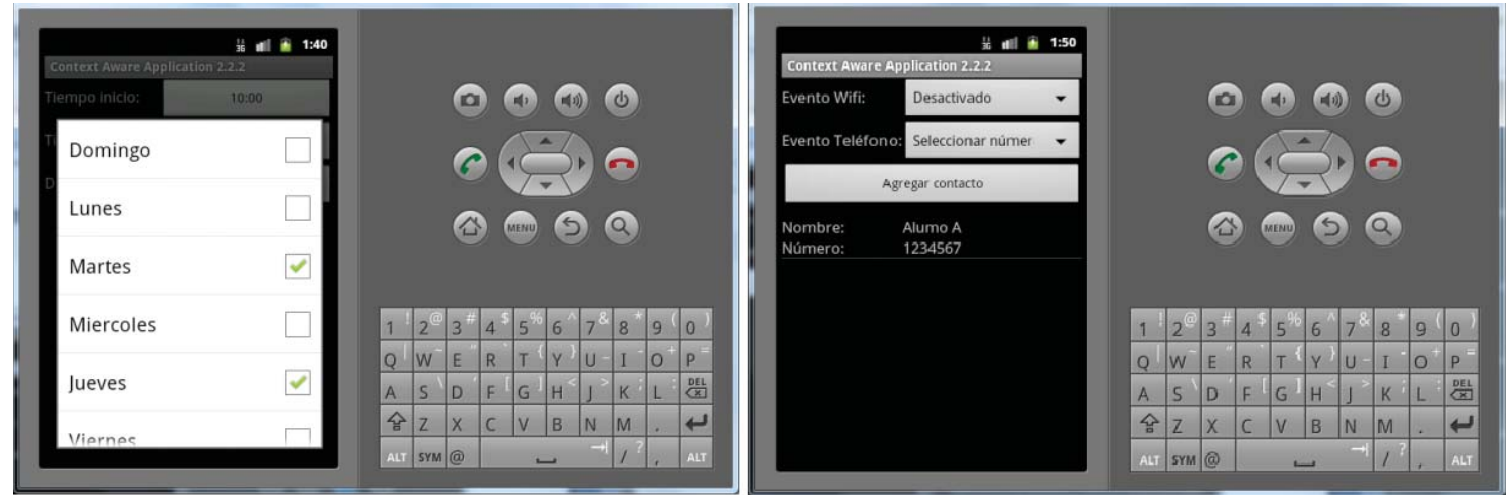

Figura 8. Configuración de reglas

Elaboración propia.

B. SQLiteManager.- Es el módulo que maneja la base de datos que registran los usuarios. Cada regla agrupa los contextos que el usuario desea registrar y asigna los eventos respectivos a la regla para todo el grupo de contextos asociados. El grupo de reacciones estará asociado a una sola regla.

C. Services.- Se accede a estos servicios desde el GUI para activarlo o desactivarlo. En el módulo se tienen dos servicios:

- ServiceTaskSensor, es la clase que registra los datos de los diferentes sensores periódicamente.

- ServiceEventTrigger, es la clase principal de la aplicación. En este servicio se inicializan todas las tablas temporales, recibe todos los parámetros de sensores obtenidos para ser validados y registrar todos los contextos principales. Uno de los problemas que se enfrentó es el tiempo de vida del servicio. Con el transcurso del tiempo, el sistema operativo destruye automáticamente los servicios que tiene ejecutándose por cierto lapso. Para evitar esto, se empleó el método 'startForeground', con el fin de lograr una prioridad superior y poder ser el último candidato que sea eliminado de la memoria. Inicializa las tablas temporales, ya que el SQLite no permite acceder a la base de datos en concurrencia.

D. DataCollectorManager.- Este servicio recibe los datos de los diferentes sensores. Se utilizó la clase ServiceTaskSensor para obtener los datos que serán enviados mediante un interface. Se encapsularon todos los sensores en una sola clase para facilitar su manejo. 
E. SimpleContextManager.-Es el motor del análisis de datos de contextos. Mantiene todas las tablas temporales, registros de datos en un archivo plano para poder ejecutarlo posteriormente en una desktop como primera prueba, y por último, validar el evento que recibe según los contextos actuales mantenidos en las tablas temporales.

F. ReactionManager.- Es el encargado de lanzar las reacciones correspondientes asociadas a una regla.

G. ANNManager.- Es el encargado de crear la red neuronal y formar el modelo para su posterior uso. Para poder culminar esta etapa, se procedió a construir una interfaz que acepte las variables de entrada descritas en el punto 6.2 para predecir la métrica de éxito que es la variable de salida descrito en el punto 6.1. Las pruebas de clasificación se efectuaron con el modelo de red neuronal artificial multicapa feed forward (Rumelhart, Hinton y Williams 1986) usando el algoritmo de aprendizaje de retropropagación de Levenberg-Marquardt (gradiente descendente optimizado). A fin de mejorar la precisión de la red, se empleó una cantidad más amplia de datos, considerando un conjunto de prueba adicional al de validación. Se probaron dieciséis arquitecturas de redes neuronales artificiales. La distribución del número de capas como la cantidad de neuronas por capa se dio bajo la permutación de los números 5, 9, 10 y 20 (obtenidos por una función random de rango [2; 20]). La función de transición empleada en la red perceptrón multicapa feed forward, es la función sigmoidal, para la capa de entrada y las capas ocultas, y una función lineal, para la capa de salida de la red. De los 13596 conjuntos de datos empleados (cada conjunto tiene cinco indicadores) en la preparación de las redes, se emplearon 12596 en el conjunto de entrenamiento, quinientos en el conjunto de validación y quinientos en el de prueba.

\subsubsection{Evaluación de la red}

El entrenamiento de las diferentes arquitecturas de redes neuronales artificiales se evaluó teniendo en consideración dos métricas: el grado de error y el rendimiento (error cuadrático medio, MSE). Para la medición del grado del error de cada arquitectura se empleó el error absoluto medio (MEAN) del conjunto de validación y del conjunto de prueba. Finalmente, la definición del mejor modelo se realizó basándose en el cálculo del error absoluto medio (MEAN) de ambos conjuntos. Para la evaluación de los resultados de salida de las diferentes arquitecturas probadas se empleó el índice de rendimiento (MSE), proporcionado por Matlab, relativo al conjunto de entrenamiento, y el error absoluto medio (MEAN) de los conjuntos de validación y prueba. Además, se calculó la desviación estándar del error absoluto de ambos conjuntos para observar la estabilidad de la red. La arquitectura que 
presenta menor error y que mejor se ajustó para la predicción del modelo es la red feed forward $20 \times 10$ (20 capas ocultas, 10 neuronas en la capa escondida, 10 neuronas en la entrada y una neurona en la salida). Por lo tanto, se escogió la mencionada red como la más apta para la predicción del modelo debido al bajo porcentaje de error que obtiene en los conjuntos de validación y prueba.

\section{Conclusiones}

Este documento propone un modelo basado en técnicas de inteligencia artificial, como el sistema basado en reglas y redes neuronales artificiales, para inferir el contexto del usuario de un dispositivo móvil, y con esto prestar una aplicación o reacción más específica o adecuada para satisfacer la necesidad del usuario, y lograr con ellos que las aplicaciones de entorno en los dispositivos móviles se optimicen. Se construyó un marco general de inferencia alternativo a los ya existentes, con el fin de proporcionar una infraestructura básica para facilitar el desarrollo de aplicaciones context-aware, mediante la integración de la información procedente del entorno.

Asimismo, se realizó una revisión de la viabilidad de las redes neuronales artificiales y del principio basado en reglas para la optimización de aplicaciones conscientes del entorno, estableciendo un proceso que utilice los datos históricos de contextos similares ejecutados por los usuarios. Los contextos son obtenidos con base en las actuaciones reales del usuario, proporcionando una manera de comparar los datos históricos del usuario usando como herramienta las redes neuronales artificiales y el principio basado en reglas.

\section{Bibliografía}

Ableson, Frank; Collins, Charlie y Robi, Sen (2010). Android, guía para desarrolladores. Madrid: Anaya Multimedia.

Bulfoni, Adolfo; Coppola, Paolo; Della Mea, Vincenzo; Di Gaspero, Luca; Mischis, Danny; Mizzaro, Stefano; Scagnetto, Ivan y Luca Vassena (2008). Al on the move: Exploiting Al techniques for context inference on mobile devices. Proc. 18th European Conf. Artificial Intelligence (ECAI 08). Ámsterdam: IOS Press.

Carretero, Noelia y Bermejo, Ana Belén (2005). Inteligencia ambiental. Madrid: Centro de Difusión de Tecnologías, Universidad Politécnica de Madrid. <http:// www.ceditec.etsit.upm.es>. [Consulta: 13 de junio de 2011]

Coppola, Paolo; Della Mea, Vincenzo; Di Gaspero, Luca; Menegon, Davide; Mischis, Danny; Mizzaro, Stefano; Scagnetto, Ivan y Luca Vassena (2010). "The context-aware browser". IEEE Intelligent Systems. Volumen 25, núm. 1, p. 11. 
Cyberstore. Los 10 mejores teléfonos inteligentes. <http://celularesdoblechip.com/ los-10-mejores-telefonos-inteligentes-smartphones.html>. [Consulta: 2 de mayo de 2011]

Díaz, Jesús; Lara, Ezequiel y Federico Mon (2009). Inteligencia ambiental en dispositivos móviles. Madrid: Facultad de Informática de la Universidad Complutense de Madrid.

Gartner, Inc. El escenario de la seguridad de la información de Gartner para el 2011. <www.biometria.gov.ar/media/45623/gutierrez_gartner.pdf>. [Consulta: 25 de abril del 2011]

IDC. Android and iOS Combine for 92.3\% of All Smartphone Operating System Shipments in the First Quarter While Windows Phone Leadpfrogs BlackBerry, According to IDC. <http://www.idc.com/getdoc. jsp?containerld=prUS24108913 $>$. [Consulta: 7 de junio del 2013].

Isasi, Pedro e Inés Galván (2004). Redes neuronales artificiales. Un enfoque práctico. Madrid: Pearson Education.

Meier, Reto (2010). Professional android 2 application development. Indianápolis: John Wiley \& Sons.

Oficina de Coordinación Nacional de Posicionamiento, Navegación, y Cronometría por Satélite. "Sistema de posicionamiento global al servicio del mundo". <http://www.gps.gov/spanish.php>. [Consulta: 4 de mayo del 2011]

Rumelhart, David; Hinton, Geoffrey y Ronald Williams (1986). Learning internal representations by error propagation, en Rumelhart, D. E.; McClelland, J. L. y el PDP Research Group. Parallel distributed processing: explorations in the microstructure of cognition. Volumen 1. Massachusetts: MIT Press.

Sarle, Warren (1994). "Neural networks and statistical models", en SAS Institute (ed.). Proceedings of the 19th Annual SAS Users Group International Conference. Cary, NC: SAS Institute, (pp.1538-1550).

Schmidt, E. (2013). Seis predicciones para nuestro futuro digital, según el director de Google. CNN. <http://cnnespanol.cnn.com/2013/04/27/seis-prediccionespara-nuestro-futuro-digital-según-el-ceo-de-google/>. [Consulta: 6 de junio del 2013]

Siau, Keng; Sheng, Hong y Fiona Nah (2004). The value of mobile commerce to customers. The Third Annual Workshop on HCl Research in MIS. Washington DC, pp. 65-69.

Tsai, Flora; Han, Wenchou; Xu, Junwei y Hock-Chuan Chua (2009). Design and development of a mobile peer-to-peer social networking application. Expert systems with applications. Volumen 36, núm. 8, pp. 11077-11087. 


\section{Glosario de términos}

- Contexto: Entorno físico o de situación, conjunto de circunstancias que condicionan un hecho. Cualquier información que caracteriza una situación entre el usuario, la aplicación y el ambiente que nos rodea.

- Clasificación: Es la técnica que se utiliza para la identificación, agrupación y distribución sistemática de ítems u objetos semejantes, con características comunes o sistema determinado y que pueden ser, con posterioridad, diferenciadas según su tipología fundamental. Dicho proceso se aplica de acuerdo a un esquema lógico predeterminado para señalar su ubicación.

- Disponibilidad de información: Acceso a la información y los sistemas de tratamiento de esta por parte de los usuarios autorizados cuando lo requieran. 